\title{
Development of Head Injury Assessment Reference Values Based on NASA Injury Modeling
}

Jeffrey T. Somers, ${ }^{1}$ John W. Melvin, ${ }^{2}$ Ala Tabiei, ${ }^{3}$ Charles Lawrence, ${ }^{4}$ Robert Ploutz-Snyder, ${ }^{5}$ Bradley Granderson, ${ }^{6}$ Alan Feiveson, ${ }^{7}$ Michael Gernhardt, ${ }^{8}$ John Patalak $^{9}$

${ }^{1}$ Wyle Integrated Science and Engineering Group

1290 Hercules Dr., Houston, Texas 77058

281-483-6010 Office

281-483-2767 Fax

jeff.somers@nasa.gov

${ }^{2}$ Tandelta, Inc.

Ann Arbor, Michigan

734-913-4826 Office

tandelta@earthlink.net

${ }^{3}$ University of Cincinnati

Cincinnati, Ohio

513-331-9139 Office

atabiei@aol.com

${ }^{4}$ NASA Glenn Research Center

21000 Brookpark Road, Cleveland, Ohio 44135

216-433-6048 Office

lawrence@nasa.gov

${ }^{5}$ Universities Space Research Association Division of Space Life Sciences

3600 Bay Area Blvd., Houston, Texas 77058

281-483-6296 Office

robert.ploutz-snyder-1@nasa.gov

\author{
${ }^{6}$ Wyle Integrated Science and Engineering Group \\ 1290 Hercules Dr., Houston, Texas 77058 \\ 281-461-2780 Office \\ brad.granderson@nasa.gov
}

${ }^{7}$ NASA Johnson Space Center, Space and Life Sciences Directorate
2101 NASA Parkway, Houston, Texas 77058
281-483-6603 Office
alan.h.feiveson@nasa.gov
${ }^{8}$ NASA Johnson Space Center, Space and Life Sciences Directorate
2101 NASA Parkway, Houston, Texas 77058
281-244-8977 Office
michael.I.gernhardt@nasa.gov

${ }^{9}$ NASCAR Research \& Development Center

7010 West Winds Blvd.

Concord, NC 28027

704-720-3141 Office

ipatalak@nascar.com

NASA is developing a new capsule-based, crewed vehicle that will land in the ocean, and the space agency desires to reduce the risk of injury from impact during these landings. Because landing impact occurs for each flight and the crew might need to perform egress tasks, current injury assessment reference values (IARV) were deemed insufficient. Because NASCAR occupant restraint systems are more effective than the systems used to determine the current IARVs and are similar to NASA's proposed restraint system, an analysis of NASCAR impacts was performed to develop new IARVs that may be more relevant to NASA's context of vehicle landing operations. Head IARVs associated with race car impacts were investigated by completing a detailed analysis of all of the 2002-2008 NASCAR impact data. Specific inclusion and exclusion criteria were used to select 4071 impacts from the 4015 recorder files provided (each file could contain multiple impact events). Of the 4071 accepted impacts, 274 were selected for numerical simulation using a custom NASCAR restraint system and Humanetics Hybrid-III $50^{\text {th }}$ percentile numerical dummy model in LS-DYNA. Injury had occurred in 32 of the 274 selected impacts, and 27 of those injuries involved the head. A majority of the head injuries were mild concussions with or without brief loss of consciousness. The 242 non-injury impacts were randomly selected and representative of the range of crash dynamics present in the total set of 4071 impacts. Head dynamics data (head translational acceleration, translational change in velocity, rotational acceleration, rotational velocity, HIC-15, HIC-36, and the Head 3ms clip) were filtered according to SAE J211 specifications and then transformed to a log scale. The probability of head injury was estimated using a separate logistic regression analysis for each log-transformed predictor candidate. Using the log transformation constrains the estimated probability of injury to become negligible as IARVs approach zero. For the parameters head translational acceleration, head translational velocity change, head rotational acceleration, HIC-15, and HIC-36, conservative values (in the lower 95\% confidence interval) that gave rise to a $5 \%$ risk of any injury occurring were estimated as $40.0 \mathrm{G}, 7.9 \mathrm{~m} / \mathrm{s}, 2200 \mathrm{rad} / \mathrm{s}^{2}, 98.4$, and 77.4 respectively. Because NASA is interested in the consequence of any particular injury on the ability of the crew to perform egress tasks, the head injuries that occurred in the NASCAR dataset were classified according to a NASA-developed scale (Classes I - III) for operationally relevant injuries, which classifies injuries on the basis of their operational significance. Additional analysis of the data was performed to determine the probability of each injury class occurring, and this was estimated using an ordered probit model. For head translational acceleration, head translational velocity change, head rotational acceleration, head rotational velocity, HIC-36, and head $3 \mathrm{~ms}$ clip, conservative values of IARVs that produced a $5 \%$ risk of Class II injury were estimated as $50.7 \mathrm{G}, 9.5 \mathrm{~m} / \mathrm{s}, 2863 \mathrm{rad} / \mathrm{s}^{2}, 11.0 \mathrm{rad} / \mathrm{s}, 30.3$, and $46.4 \mathrm{G}$ respectively. The results indicate that head IARVs developed from the NASCAR dataset may be useful to protect crews during landing impact. 